\title{
A New Method of Visual Recognition for Weld Appearance
}

\author{
Wei Xian*
}

Engineering Technology Department, Panzhihua University, Panzhihua, Sichuan, China

\begin{abstract}
The visual recognition methods of texture feature of welding seam have the important significances to realize the automatic tracking of welding seam in the steps of subsequent welding and cover welding of multi-layer welding. Starting from the point of view of making full use of texture feature of welding seam image, the method research and algorithm design are respectively carried out on the 2 steps (image processing and position identification) of visual recognition of welding seam. In the image processing, the $\pm 45^{\circ}$ Sobel operators are used to carry out the edge detection. In the position identification, the projection method and the regional integration method are combined so as to determine the position of middle lines of welding seams. The result shows that the proposed method is simple and easy in principal and can, at the same time, meet the timeliness and accuracy requirement.
\end{abstract}

Keywords: Image processing, regional integration, texture of welding seam, visual recognition.

\section{INTRODUCTION}

In order to realize the automation and intelligentization of welding, it is required that the automatic identification and accurate positioning should be carried out on the subsequent welding area. In the process of subsequent welding and cover welding of large heavy plate welding, the automatic welding devices should be able to timely identify the specific position of welding seam so as to obtain the offset of welding torch relative to the middle line of welding seam, control the machine to make the corresponding adjustment and realize the automatic tracking of welding seam $[1,2]$.

The traditional visual identification usually adopts the active visual method of structured light or laser scanning to illuminate the line structured light or laser beam on the structures of distinct spatial geometry so as to form the linear lights. Because of the changes of surface depth of object, light distortion and discontinuousness, the images collected by the camera show the evident geometry features. Through the image analysis, the 3D structure information [3] of groove can be obtained from them. However, different with the groove weld detection, the structured light (or laser scanning) methods have certain limitations on the visual identification of welding seam because the welding seams have no apparent structure features in the direction of depth. Aiming at the drawbacks of traditional methods, the thoughts of making full use of texture feature of welding seam are put forward. Under the environmental light, the CCD camera is used to photograph so as to obtain the image of welding seam, wherein the portions of welding seam have recurring regular textures, while the shape and randomness of parent material portions are evidently different with the

*Address correspondence to this author at the Engineering Technology Department, Panzhihua University, Panzhihua, Sichuan, China;

Tel: 18982355000; E-mail: 110801844@qq.com characteristics showed by the texture of welding seams de spite of interference information of workpiece surface rusting etc. The described method is firstly to strengthen the textures through the image processing, and then make use of the image analysis method to distinguish the welding seam texture and the parent material so as to determine the middle line position of welding seams.

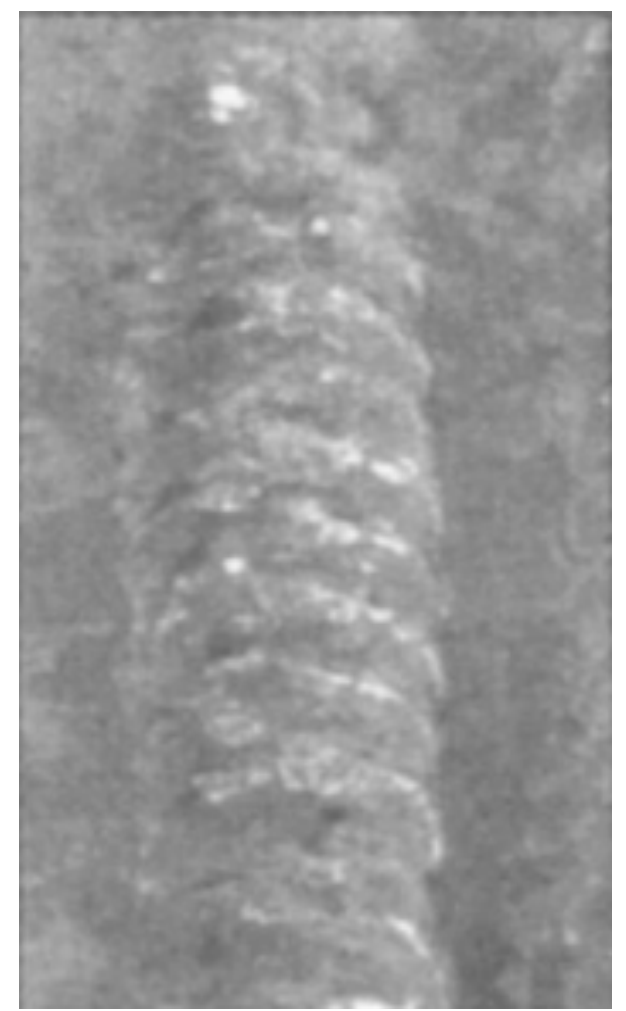

Fig. (1). Original image of weld. 


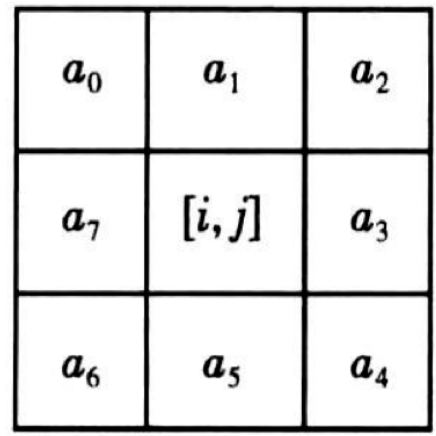

(a) $3 \times 3$ territory of dot $[i, j]$

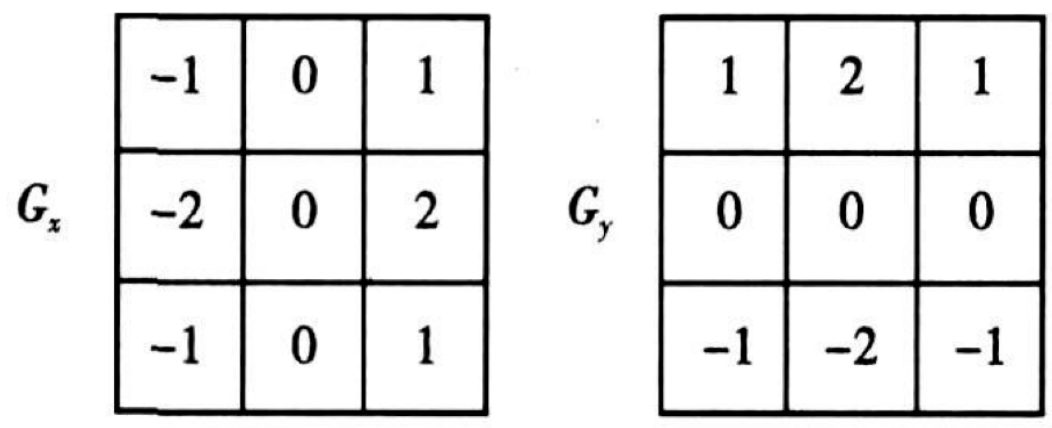

(b) the convolution masks of horizontal direction and vertical direction


(c) the convolution masks of $\pm 45^{\circ}$ direction

Fig. (2). Convolution masks.

\section{THE IMAGE PROCESSING BASED ON SOBEL EDGE DETECTION}

Through the observation of original image of welding seams (photo 1), it can be learned that the surface morphology of welding seam shows the fish scalelike wave on the gray-scale images. This texture feature is a reliable basis to distinguish the welding seam and the parent materials. So an important purpose that should be reached by the steps of image processing is to extract the image information of welding seam from the background information of parent materials and strengthen the texture features of welding seam to the maximum extent.

In the gray-scale image, the area where the textures of welding seams change greatly corresponding to the gray value, namely the area where the gradient of gray is big, can be found out by the edge detecting method. As an approximate method of gradient calculation, Sobel operators are the commonly used operators of edge detection [4]. As shown in

Fig. (2a), the $a_{0} \sim a_{7}$ dot arrays within the $3 \times 3$ territory are respectively the gray values of dot $[i, j]$ territory pixel. Thus, the partial derivative of gray in the dot $[i, j]$ can be obtained by calculation, i.e

$$
\begin{aligned}
& \left.y^{\prime} f_{x}=\left(a_{2}+2 a_{3}+a_{4}\right)\right)-\left(a_{2}+2 a_{7}+a_{6}\right) \\
& y^{\prime} f_{y}=\left(a_{0}+2 a_{1}+a_{2}\right)-\left(a_{6}+2 a_{5}+a_{4}\right)
\end{aligned}
$$

The above 2 partial derivatives can be realized with convolution masks. Every dot in the image can use the 2 masks as shown in Fig. (2b) to carry out the convolution. The response of convolution mask in the horizontal direction $G_{x}$ are the largest to the horizontal edges. And the response of convolution mask in the vertical direction $G_{y}$ are the largest to the vertical edges.

Considering that the texture shape turns the shape of approximate semi-ellipse arc, Sobel convolution masks of $\pm 45^{\circ} \mathrm{G} 1, \mathrm{G} 2$ are selected to carry out the edge detection (the 2 responses of edge are the largest respectively to $+45^{\circ}$ direction and $-45^{\circ}$ direction). And the edge points that have the bigger gray gradient along any direction of them can be obtained, which just can highlight the circular part of 




Fig. (3). Result of edge detection.

welding seam texture in the direction of both sides, and reduce, to certain extent, the interference of miscellaneous dots that have no direction in the parent materials. The results of edge detection are as shown in Fig. (3).

From the results, except a small amount of noise dots, other informations irrelevant with welding seam texture have been filtered out. Comparing with the original image, it can be found that the discontinuous dots and lines fundamentally reflect the profiles of fish scalelike textile, and the interferences generated by the parent materials are very less, which fundamentally reaches the purposes of image processing and provides for the subsequent steps the images that can be analyzed easily.

\section{THE PROJECTION METHOD AND THE RE- GIONAL INTEGRATION OF POSITION IDENTIFI- CATION OF WELDING SEAMS}

The images are processed and analyzed so as to extract the shape characteristics of welding seam texture. After the binary Figures (Fig. 3) are obtained, the further characteristics analysis needs to be carried out so as to determine the correct position of welding seams. Specifically, the coordinate of the middle line of welding seam in the image is found starting from the result of image processing. The control mechanism will make the corresponding adjustment according to the distances between this result and the target position so as to make the welding torch always move forward along the correct path. Because the tracking of welding seam is required to have accuracy and timeliness at the same time, the research adopts to design the algorithm the combination thought between the projection method that the algorithm is simple, but the accuracy is not stable, and the regional inte-

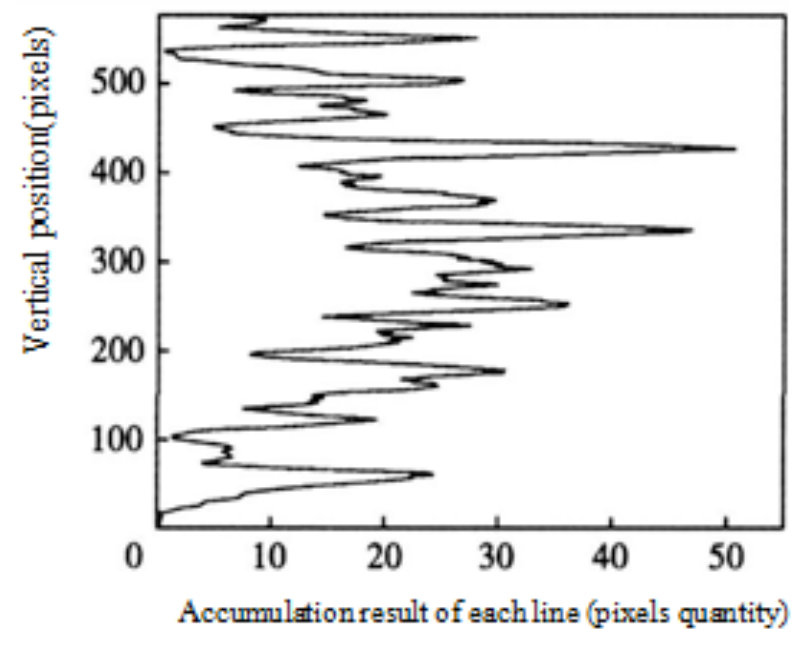

(a) the result of horizontal projection

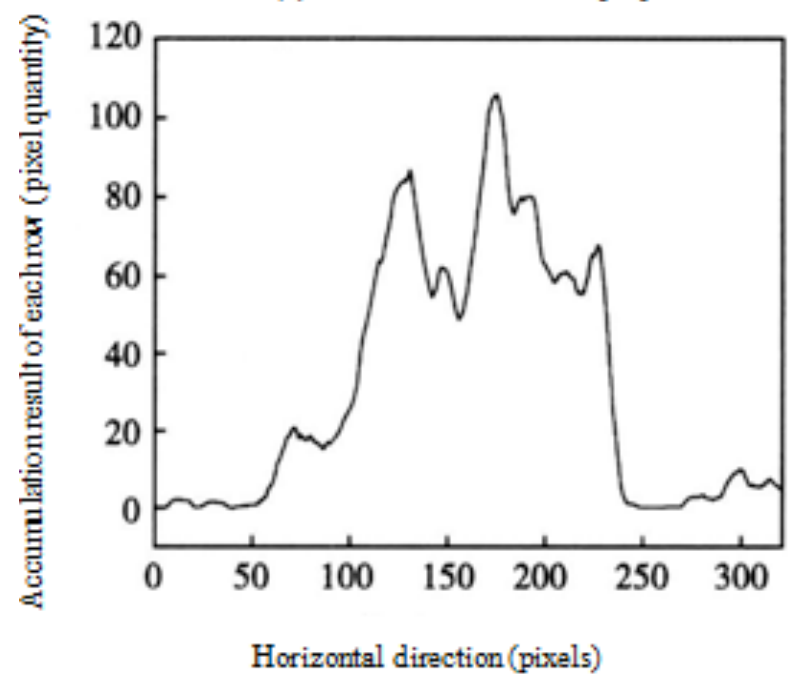

(b) the result of vertical projection

Fig. (4). Projections in horizontal and vertical directions.

gration method that the positioning is correct, but the consumed time is relatively longer.

\subsection{The Projection Method is Used to Carry Out the Pre- liminary Position Identification}

The projection is carried out on the binary figures that will be identified in both horizontal and vertical direction, namely, the quantity of pixels of value 1 is statistically accumulated line by line and row by row so as to obtain the distribution density situation of pixels of value 1 in the image [5]. This distribution is as shown in Fig. (4). Fig. (4a) is the accumulation result of each line, and Fig. (4b) is the accumulation result of each row.

From the result of vertical projection, it can be seen that the textures of welding seam mainly appear in the middle and lightly left position, while, in the projection image of horizontal direction, the wave peak appears in almost equal intervals. These reflect the rules of periodicity distribution of texture.

Through the analysis of 2-direction projection, the searched region of welding seam texture can be reduced so 


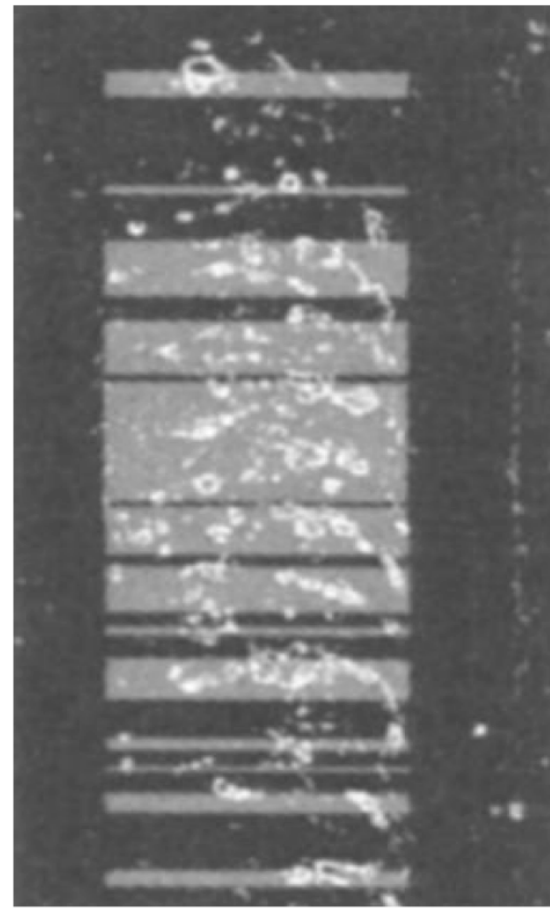

Fig. (5). Candidate areas.



Fig. (6). Overlap of texture and crescent shape.

as to reduce the subsequent calculation and enhance the efficiency of algorithm. The gray area in Fig. (5) is the candidate region to search the texture of welding seam.

\subsection{The Crescent 0 Region Integration is Used for the Precise Positioning}

After the region of texture of welding seams is preliminarily determined by the projection method, the regional integration method can be used to precisely position the

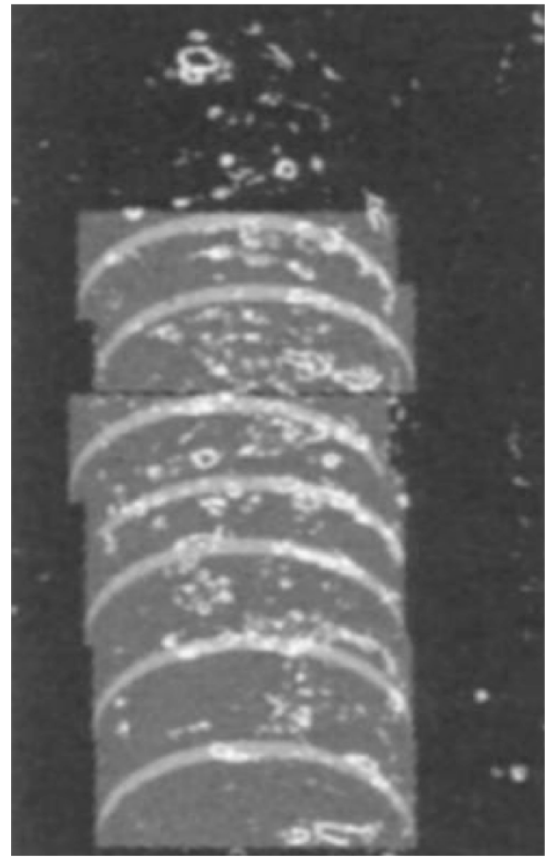

Fig. (7). Schematic of certain textures.

stripe curve texture so as to obtain the accurate position of welding seams.

In Fig. (3), the textures consist of the intermittent dots and lines. And the whole shapes formed by these intermittent dots and lines are approximately the semi-ellipse arc. In order to detect a texture, a crescent-shaped region (Fig. 6) can be constructed and moved in the image. The integration is done in this region. When a texture is included in this region or, it can be said, when the texture overlaps with this region), the result value of regional integration is very large. Accordingly, the position of texture can be obtained.

Fig. (7) is the result of texture identification. In order to simplify the algorithm, the following 2 characteristics of texture need to be used: (1), a certain interval of distance exists in every 2 adjacent textures; (2), the center of each texture is roughly in the same line. And this line is basically along the vertical direction.

From the characteristic (1), it can be learned that, under the condition that the first texture is found out, there is no need to proceed the line-by-line search in order to find the $2^{\text {nd }}$ texture. And several pixel lines can be skipped, and then the search can begin again; and the characteristic (2) determine that the lateral position of subsequent texture can only possibly change within the very small range near to the lateral position of previous texture, which means, in the positioning process of each texture, there are the prior informations of previous texture position, and the pixel-by-pixel search and the regional integration can only be carried out within very small range. The calculation is reduced greatly.

After many textures are determined, through the linear fitting to each center point, the linear equation that shows the middle line position of welding seams will be obtained. If all the straight lines are drawn in the original image of welding seam, the result diagrammatic sketch of identification method of this welding seam will be got, as shown in Fig. (8). 


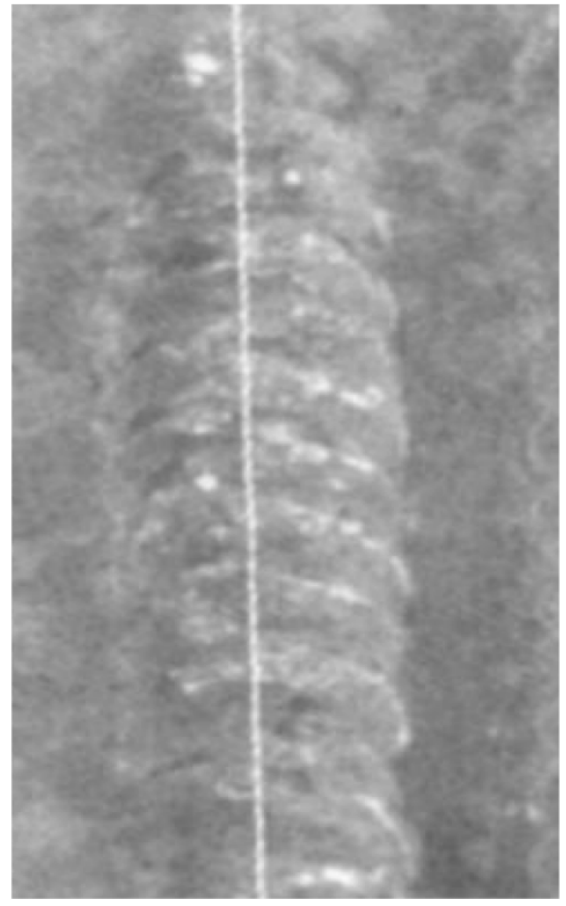

Fig. (8). Location of central line located.

\section{EXAMPLE VERIFICATION AND COMMENT ON ACCURACY AND TIMELINESS}

\subsection{Experimental Platform and Experimental Conditions}

Matlab 6.5 is used as the calculation platform. And the procedures are written to carry out the feasibility verification [6]. The processed image is as shown in Fig. (1). The relevant welding parameter and image parameter include: welding torch speed of advance: $500 \mathrm{~mm} / \mathrm{min}$, size of intercepted image of welding seams: 321 pixelX575 pixel, and one millimeter corresponds to 15 pixels. The computer for example verification is Pentium4 CPU, the host frequency is $2.40 \mathrm{GHz}$ and the RAM is $1 \mathrm{G}$.

\subsection{Comment on the Accuracy and Timeliness of Identi- fication Result}

The middle line positions of welding seam identified by algorithm are compared with the real position of welding seam and the error is within 10 pixels. As shown in Fig. (8), the accuracy of welding seam identification has reached the satisfactory result.

The timeliness is the key indicator to measure if the algorithm can be applied to the process of actual automatic welding processing. The conditions that the speed of image processing needs to meet in algorithm include: The processing time of a single image should be less than or equal to the result that the welding region length covered by the image is divided by the welding speed. To sum up various conditions, it can be learned after calculations that the processing time of a single image must be controlled within $4 \mathrm{~s}$. With the above computer as platform, after the test, the running time that the procedure of algorithm realization through Matlab processes an image of welding seam is about $2.7 \mathrm{~s}$.

Considering that the "for" cycle or "for" cycle nesting are inevitably used in many places in the writing of Matlab programs and this grammatical structure makes the running speed of Matlab program reduced greatly; considering, in the actual application, the $\mathrm{C}$ code is written on DSP platform so as to carry out the calculation of all the image processing and the running of $\mathrm{C}$ code will be more fluent comparing with Matlab program, it can be judged that the identification method of welding seam described in this article can meet the requirement of timeliness.

\section{RESULT}

(1) Through Sobel edge detection, the image preprocessing is carried out and the binaryzation texture image with less miscellaneous dots can be obtained.

(2) The crescent integration masks adapting to the target image are constructed. And the regional integration method can be used to complete the position identification of welding seam texture element. The accuracy is within $1 \mathrm{~mm}$.

(3). The time that algorithm processes an image under the calculation environment of Matlab is about $2.7 \mathrm{~s}$, which can meet the requirement of accuracy after analysis.

\section{CONFLICT OF INTEREST}

The authors confirm that this article content has no conflicts of interest.

\section{ACKNOWLEDGEMENTS}

Declared none.

\section{REFERENCES}

[1] L. Xueqin, K. Zhang, and W. Yixiong, "Condition and expectation of automatic weld seam tracking system", Chinese Journal of Mechanical Engineering, vol. 39, no.12, pp. 80-85, 2003.

[2] G. Xiangdong, L. Mingtao, and C. Jianhui, "Applicat ion of vision sensing and image processing technique in welding control", Modern Welding, vol. 11, pp. 15- 18, 2006.

[3] Z.G. Jun, "Machine Vision", Science Press: Beijing, 2005.

[4] Z.Y. Jin, "Image Processing”, Tsinghua University Press: Beijing, 2005.

[5] Z. Y Jin, "Image Analysis", Tsinghua University Press: Beijing, 2005.

[6] R.C. Gonzalez, R.E. Woods, and S.L. Eddins, "Digital image processing using Matlab", Pearson Prentice Hall: Upper Saddle River 2004 